\title{
Zur Kenntnis von Harzbestandteilen
}

\author{
Zweite Mitteilung: \\ Über Bestandteile der Sumatrabenzoe
}

Von

Hans Lieb und Alois Zinke

Aus dem Medizinisch-chemischen und dem Chemischen Institut der Universität Graz

(Vorgelegt in der Sitzung am 10. Jänner 1918)

In unserer ersten Mitteilung ${ }^{1}$ über das Siaresinol aus Siambenzoe konnten wir zeigen, daß dieser Verbindung die Formel $\mathrm{C}_{30} \mathrm{H}_{48} \mathrm{O}_{4}$ zukommt. Wir sprachen die Vermutung aus, daß das von Lüdy aus der Siambenzoe isolierte Benzoresinol $^{2}$ mit dem Siaresinol Reinitzer's ${ }^{3}$ identisch sei. Da Lüdy behauptet, daß das von ihm aus der Sumatrabenzoe isolierte Resinol ${ }^{4}$ auch mit dem von ihm aus der Siambenzoe dargestellten identisch ist, waren wir, um eine sichere Entscheidung fällen zu können, bemüssigt, auch die Sumatrabenzoe in den Kreis unserer Untersuchungen zu ziehen.

Obwohl wir bei der Aufarbeitung des Sumatrabenzoeharzes im wesentlichen nach den Vorschriften Lüdy's arbeiteten, zeigten sich hierbei schon bedeutende Unterschiede gegenüber dem Verhalten bei der Isolierung des Resinols aus der Siambenzoe. Während nämlich diese in heißer, verdünnter Natron-

1 Mon. f. Ch., 1918.

2 Arch. d. Pharm., 231, $46 !$ (1893).

3 Ibidem, 252, 341 (1914).

4 Ibidem, 231, 43 (1893). 
lauge nicht vollständig löslich ist, sondern das Siaresinol als Natriumsalz ungelöst bleibt, löst sich die Sumatrabenzoe bis auf die holzigen Verunreinigungen vollständig auf. Bei einstündigem Kochen der filtrierten alkalischen Lösung scheiden sich allmählich weiße Nadeln $a b$, die sich als die Natriumverbindung eines neuen, von Lüdy nicht aufgefundenen Resinols erwiesen. Da diese Verbindung und ihr Natriumsalz die Ebene des polarisierten Lichtstrahles nach links drehen, nemnen wir sie $l$-Benzoresinol.

Beim Versetzen der alkalischen Mutterlauge mit wenig Ather (nach Lüdy's Vorschrift), scheidet sich ein zweites, krystallisiertes Natriumsalz einer Verbindung ab, die in ihren Eigenschaften mit Ausnahme des Schmelzpunktes und der Zusammensetzung mit Lüdy's Benzoresinol übereinstimmt. Da diese Verbindung sich als rechtsdrehend erwies und ihr auch, wie wir weiter unten zeigen, eine andere Formel zukommt, bezeichnen wir sie als $d$-Sumaresinol.

Lüdy stellt für sein Benzoresinol, das nach ihm bei $274^{\circ}$ unter weit früherem Sintern schmilzt, die Molekularformel $\mathrm{C}_{16} \mathrm{H}_{26} \mathrm{O}_{2}$ auf. $\mathrm{Er}$ beschreibt einen Methyl-, Äthyl- und Isobutyläther, sowie ein durch Wasser leicht hydrolysierbares, krystallisiertes Kaliumsalz. Das freie Resinol ist nach Lüdy leicht löslich in Natronlauge, Kalilauge, Ammoniak und in den meisten organischen Lösungsmitteln. Die Lösungsfarbe in konzentrierter Schwefelsäure ist karminrot.

Das von uns analog erhaltene $d$-Sumaresinol löst sich übereinstimmend mit Lüdy's Verbindung leicht in verdünnten, heißen Lösungen von Natronlauge, Kalilauge, Natriumcarbonat, Ammoniak, sowie in den meisten organischen Lösungsmitteln. Es krystallisiert auch aus verdünntem Methylalkohol, Äthylalkohol und Aceton in Nadelbüscheln. Abweichend ermittelten wir den Schmelzpunkt der reinen Verbindung mit $298^{\circ}$ bis $299^{\circ}$ (unkorr.). Zahlreiche Analysen sowie Molekulargewichtsbestimmungen des freien $d$-Sumaresinols, wie auch von Deriraten desselben lassen es unzweifelhaft erscheinen, daß dieser Verbindung die Formel $\mathrm{C}_{30} \mathrm{H}_{48} \mathrm{O}_{4}$ zukommt. Die Lösungsfarbe in konzentrierter Schwefelsäure ist orangerot. Trotz dieser abweichenden Eigenschaften sind wir überzeugt, daß Lüdy's 
Benzoresinol im wesentlichen identisch ist mit unserem $d$-Sumaresinol, jedoch dürfte seine Verbindung, wie wir weiter unten darlegen werden, verunreinigt gewesen sein durch das von uns aufgefundene $l$-Benzoresinol.

Unser $d$-Sumaresinol hat dieselbe Zusammensetzung wie das Siaresinol aus der Siambenzoe, stimmt jedoch in seinen Eigenschaften, wie Schmelzpunkt, optisches Verhaiten, Lösungsfarbe in konzentrierter Schwefelsäure und Löslichkeit der freien Veabindung, wie des Natriumsalzes, mit jenem nicht überein. Es ist deshalb als ein Isomeres des Siaresinols zu betrachten.

Das aus verdünntem Methylalkohol krystallisierte $d$-Sumaresinol enthält im lufttrockenen Zustand 1 Molekül Krystallwasser. Das Natriumsalz ist leicht hydrolysierbar und deshalb nicht absolut rein zu erbalten. Wegen der leichteren Filtrierbarkeit verwendet man zum Umkrystallisieren des Natriumsalzes zweckmäßiger eine Natriumcarbonatlösung an Stelle der Natronlauge. Das Salz krystallisiert mit 4 Molekülen Krystallwasser.

Die von uns zur sicheren Festlegung der Formel dargestellten Methyl- und Äthyläther wurden über das in reinem Zustande nicht isolierte Silbersalz erhalten. Beide zeigen wesentlich höhere Schmelzpunkte als die entsprechenden Verbindungen Lüdy's und enthalten eine Alkoxylgruppe. Aus Eisessig konnte das Resinol nicht krystallisiert erhalten werden.

Aus Mangel an Ausgangsmaterial konnten wir das l-Benzoresinol noch nicht mit der gewünschten Genauigkeit studieren. Es war uns weder möglich, die freie Verbindung krystallisiert zu erhalten, noch mit Ausnahme des Natriumsalzes analysenreine Derivate herzustellen. Einstweilen wollen wir deshalb unsere Angaben über die Zusammensetzung dieser Verbindung noch mit Vorbehalt wiedergeben. Aus den Analysen des krystallisierten, lufttrockenen und getrockneten Natriumsalzes, sowie aus den Analysen des amorphen, freien $l$-Benzoresinols erscheint die Formel $\mathrm{C}_{29} \mathrm{H}_{44} \mathrm{O}_{4}$ wahrscheinlich. Der Schmelzpunkt des Resinols liegt bei 339 bis $341^{\circ}$. Das Natriumsalz zeigt schwache optische Linksdrehung und wird von kaltem und heißem Wasser nicht 
gelöst. Die Lösungsfarbe in konzentrierter Schwefelsäure ist über gelb, orange, braunrot ein intensives Blutrot mit. schwacher Fluoreszenz.

Mischt man $l$-Benzoresinol mit $d$-Sumaresinol, so ist die Lösungsfarbe des Gemenges in konzentrierter Schwefelsäure rein karminrot, also dieselbe Farbe, wie sie Lüdy für sein Benzoresinol angibt.

Ein weiterer Beweis dafür, daß Lüdy's Resinol ein Gemisch der zwei von uns gefundenen Resinole sein, mub, ist die Tatsache, daß die Mischung letzterer sich bei der Schmelzpunktsbestimmung fast analog den Angaben Lüdy's verhält, nämlich Sintern von $260^{\circ}$ an und Schmelzen um $280^{\circ}$. Die nicht volle Übereinstimmung ist dadurch erklärlich, daß ja das Mischungsverhältnis der beiden Resinole in unserem willkürlichen Gemenge nicht dasselbe sein muß wie in Lüdy's Benzoresinol.

Über die Natur des Wasserstoffatoms, das die sauren Eigenschaften der beiden Resinole bedingt, sprechen wir dieselben Vermutungen aus wie beim Siaresinol. ${ }^{\top}$ Das saure Wasserstoffatom des $d$-Sumaresinols könnte auch wegen der leichten Löslichkeit in Sodalösung und in Ammoniak einer Carboxylgruppe angehören.

Die genauere Entscheidung dieser Frage behalten wir uns einer späteren Mitteilung vor. Wahrscheinlich wird sich auch eine Anderung in der Nomenklatur dieser Verbindungen als notwendig erweisen.

Schließlich möchten wir auf die Tatsache hinweisen, daß unter Harzbestandteilen öfters Verbindungen mit 30 C-Atomen vorkommen. Wir verweisen auf die Amyrine der Elemiharze ${ }^{2}$ und auf die von uns jetzt festgestellte Zusammensetzung des Siaresinols und des $d$-Sumaresinols.

An dieser Stelle wollen wir noch besonders hervorheben, daß die rasche Durchführbarkeit und exakte Aufklärung der Zusammensetzung der beschriebenen Verbindungen, die nur durch zahlreiche Analysen möglich war, bei den geringen

1 Mon. f. Ch., 1918.

? Biochemisches Handlexikon, Bd. 7, p. 727 bis 731 . 
Waterialmengen, die uns zur Verfügung standen, ohne die vorzüglichen mikrochemischen Methoden Pregl's undurchführbar gewesen wäre.

Unsere Untersuchungen werden fortgesetzt.

\section{Experimenteller Teil.}

Darstellung der Natriumsalze des $l$-Benzoresinols und des d-Sumaresinols.

$50 \mathrm{~g}$ gepulverte Sumatrabenzoe ${ }^{1}$ wurden mit $250 \mathrm{cmin}^{3}$ Ather ausgekocht, wobei der größte Teil des Harzes in Lösung ging. Die filtrierte Lösung wurde eingedampft und der Rückstand in sechsprozentiger Natronlauge durch Behandeln am siedenden Wasserbade gelöst. Die alkalische Lösung wurde dann zum Sieden erhitzt. Während des Kochens scheidet sich das $l$-Benzoresinolnatrium in prismatischen Nadeln aus. ${ }^{2}$ Nach einstündigem Kochen wurde heiß filtriert, das Salz mit sechsprozentiger Lauge und dann gut mit Wasser gewaschen.

Das nach dem völligen Erkalten mit etwas Äther versetzte alkalische Filtrat wurde längere Zeit stehen gelassen, wobei das Natriumsalz des $d$-Sumaresinols in feinen Nadeln auskrystallisierte, die abfiltriert und gut mit sechsprozentiger Lauge gewaschen wurden.

\section{l-Benzoresinolnatrium.}

Das in kaltem Wasser unlösliche $l$-Benzoresinolnațium wird durch siedendes Wasser, ohne gelöst zu werden, hydrolysiert, wobei das Wasser alkalische Reaktion annimmt. In Methyl- und Äthylalkohol ist das Salz leicht löslich, in Aceton auch beim Kochen nicht vollständig. Es krystallisiert aus kon-

1 Zur Verwendung kam ein vor etwa 20 Jahren von Gebe \& Co. in Dresden bezogenes Harz aus der Sammlung des botanischen Instituts der Technischen Hochschule in Graz. Für die Uberlassung des jetzt nicht beschattbaren Ausgangsmaterials sind wir Herm Prof. Fr. Reinitzer zu größtem Dank verpflichtet.

2 Zum selben Ergebnis kommt man, wenn das aus Natronlauge umgefällte und mit viel Wasser ausgekochte Rolbharz direkt mit Natronlauge verarbeitet wird. 
zentrierter, mit wenig Wasser versetzter alkoholischer Lösurg in weißen Nadeln, die 10 Moleküle Krystallwasser enthalten.

Analysen des lufttrockenen Salzes:

1. $4 \cdot 282 m g$ Substanz gaben $8 \cdot 135 \mathrm{mg} \mathrm{CO}_{2}$ und $3 \cdot 63 m g \mathrm{H}_{2} \mathrm{O}$.

2. $4.385 \mathrm{ng}^{2}$ Substanz gaben $8.31 \mathrm{ng} \mathrm{CO}_{2}$ und $3.52 \mathrm{mg} \mathrm{H}_{2} \mathrm{O}$.

3. $5 \cdot 880 \mathrm{mg}$ Substanz gaben $0.565 \mathrm{mg}$ Natriumsulfat.

4. $5 \cdot 618 \mathrm{ng}$ Substanz verloren beim Trocknen im Vakum bei $123^{\circ}$ $1 \cdot 473 \mathrm{mg} \mathrm{H}_{2} \mathrm{O}$.

In 100 Teilen:

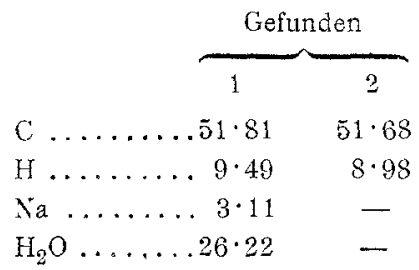

Berechnet für

$$
\mathrm{C}_{29} \mathrm{H}_{43} \mathrm{O}_{4} \mathrm{Na}+10 \mathrm{H}_{2} \mathrm{O}
$$

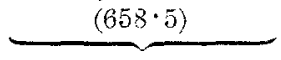

$52 \cdot 85$

$9 \cdot 64$

$3 \cdot 49$

$27 \cdot 35$

Analyse der getrockneten (äußerst hygroskopischen) Substanz:

$4.145 \mathrm{mg}$ Substanz gaben $10.99 \mathrm{mg}_{\mathrm{s}} \mathrm{CO}_{2}$, und $3.30 \mathrm{mg} \mathrm{H}_{2} \mathrm{O}$.

In 100 Teilen:

Berechnet fïr
C $\ldots \ldots \ldots, 72 \cdot 31$
H....... $8 \cdot 91$
$\underbrace{\text { Gefunden }}$
$\underbrace{\mathrm{C}_{29} \mathrm{H}_{43} \mathrm{O}_{4} \mathrm{Na}(478 \cdot 3)}$
$72 \cdot 76$
$9 \cdot 06$

Bestimmung der spezifischen Drehung im Mikropolarisationsapparat nach Emil Fischer:

Lösungsmittel: abs. Äthylalkohol.

$$
\begin{array}{lrl}
p & =2.014 & \alpha_{D}^{9 \circ}=-0.15^{\circ} \\
d=0.7993 & {[\alpha]_{D}^{9 \circ}=-9.32^{\circ}} \\
c=1.610 & & \\
l=100 \mathrm{~mm} & &
\end{array}
$$

\section{l-Benzoresinol.}

Zur Darstellung des freien $l$-Benzoresinols fällt man die alkoholische Lösung seines Salzes mit verdünnter Salzsäure, wobei sich die Verbindung als weiße Gallerte abscheidet. Um sie filtrierbar $z u$ machen, erwärmt man kurze Zeit am siedenden 
Wasserbade, wobei sie sich zu Flocken zusammenballt. Das gut mit Wasser gewaschene, bei $110^{\circ}$ getrocknete $l$-Benzoresinol ist ein weißes Pulver, das bei 339 bis $341^{\circ}$ (unkorr.) schmilzt, indem es bei $329^{\circ}$ zusammensintert und sich braun färbt. Es ist in den meisten organischen Lösungsmitteln schwer Iöslich. In Methyl- und Äthylalkohol löst es sich langsam auf, indem es vorher aufquillt. In Alkalien ist es unlöslich. In konzentrierter Schwefelsäure löst es sich in der Kälte gelb, beim schwachen Erwärmen schlägt die Farbe zuerst in ein intensives Braunrot mit schmutziggrüner Fluoreszenz, dann in Blutrot um. Bei der Liebermann'schen Cholestolprobe färbt sich die Schwefelsäure beim Zutropfen vorübergehend violett. Beim Umschütteln wird die Flüssigkeit grün, bei weiterem Zusatz von Schwefelsäure braunrot. Bei der SalkowskyHesse'schen Probe färbt sich die Schwefelsäure zuerst orangegelb, dann blutrot mit gelbgrüner Fluoreszenz, das Chloroform bleibt fast farblos. Beim Eindunsten einer methylalkoholischen Lösung in der Eprouvette wurde die Substanz einmal in sehr geringer Menge krystallisiert erhalten.

Analysen der bei $130^{\circ} \mathrm{im}$ Vakuum getrockneten, amorphen Substanz:

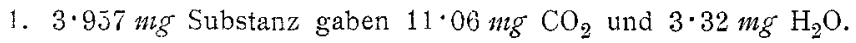

2. $4 \cdot 206 \mathrm{mg}$ Substanz gaben $11 \cdot 755 \mathrm{mg} \mathrm{CO}_{2}$ und $3 \cdot 64 m g \mathrm{H}_{2} \mathrm{O}$.

3. t.070 $n g$ Substanz gaben $11.38 \mathrm{ng} \mathrm{CO}_{2}$ und $3 \cdot 50 \mathrm{mg} \mathrm{H}_{2} \mathrm{O}$.

In 100 Teilen:

$$
\begin{aligned}
& \text { Gefunden Berechnet für } \\
& \overbrace{123}^{2} \quad \underbrace{\mathrm{C}_{29} \mathrm{H}_{41} \mathrm{O}_{4}(456 \cdot 3)}_{76 \cdot 20} \\
& \text { C......76.23 } \quad 76.22 \quad 76 \cdot 26 \quad 76 \cdot 26
\end{aligned}
$$

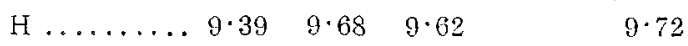

Bestimmung der spezifischen Drehung im Mikropolarisationsapparat nach Emil Fischer:

Lösungsmittel: abs. Alkohol.

$$
\begin{array}{ll}
p=1.540 & \alpha_{D}^{\sigma^{\circ}}=-0.16^{\circ} \\
d=0.807 & \alpha_{D}^{\tau^{\circ}}=-0.08^{\circ} \\
c=1.242 & {[\boldsymbol{\alpha}]_{D}^{7 \circ}=-12.88^{\circ}} \\
l=100 \mathrm{~mm} & \\
l=50 \mathrm{~mm} &
\end{array}
$$




\section{$d$-Sumaresinolnatrium.}

Das $d$-Sumaresinolnatrium ist in kaltem Wasser löslich. Die Lösung trübt sich jedoch sofort infolge Hydrolyse, weshalb ein gründliches Auswaschen des Salzes mit Wasser unmöglich ist. Zur Reinigung des Salzes verfährt man folgendermaßen: Das Salz, wie es bei der Darstellung aus dem Harze resultiert, wird abfiltriert und mit Natronlauge gut gewaschen. Dann giebt man so lange Wasser auf, bis das gesamte Salz in Lösung gegangen ist. Das wässerige Filtrat versetzt man mit soviel konzentrierter Natriumcarbonatlösung, daß die Flüssigkeit zirka sechsprozentig wird. Durch Aufkochen bringt man das durch Hydrolyse ausgeschiedene $d$-Sumaresinol in Lösung, filtriert und versetzt das Filtrat nach dem Erkalten mit wenig Äther. Das $d$-Sumaresinolnatrium scheidet sich fast augenblicklich in rein weißen Nadeln aus. In Alkohol ist das Salz leicht löslich.

Zur Analyse wurde ein mehrmals auf oben angegebenem Wege umkrystallisiertes Salz dreimal mit Eiswasser gewaschen und dann an der Luft getrocknet.

1. $5.948 \mathrm{mg}$ Substanz gaben $0.730 \mathrm{mg} \mathrm{Na}_{2} \mathrm{SO}_{4}$.

2. $4.220 \mathrm{mg}$ Substanz verloren beim Trocknen im Vakuum hei $150 \mathrm{c}$ $0.561 \mathrm{mg} \mathrm{H}_{2} \mathrm{O}$.

In 100 Teilen:

\begin{tabular}{|c|c|c|}
\hline & Gefunden & $\mathrm{C}_{30} \mathrm{H}_{4 i} \mathrm{O}_{4} \mathrm{Na}+4 \mathrm{H}_{2} \mathrm{O}$ \\
\hline $\mathrm{Na}$ & $3 \cdot 97$ & $4 \cdot 06$ \\
\hline $4 \mathrm{H}_{2} \mathrm{O} \ldots \ldots$ & $13 \cdot 30$ & $12 \cdot 72$ \\
\hline
\end{tabular}

\section{$d$-Sumaresinol.}

Beim Ansäuern einer alkalischen Lösung des $d$-Sumaresinolnatriums mit verdünnter Salzsäure wird das freie $d$-Sumaresinol in weißen, amorphen Flocken ausgefällt. Der Niederschlag wird abgesaugt, gut mit Wasser gewaschen und bei $110^{\circ}$ getrocknet. Das amorphe $d$-Sumaresinol ist ein weißes Pulver, das in Methyl-, Äthylalkohol, Äther, Aceton, Eisessig, Chloroform schon in der Kälte, in Benzol und Xylol erst in der Hitze leicht löslich ist. Von Ligroin wird es nicht 
gelöst. Aus alkoholischen Lösungen, die mit Wasser bis zur Trübung versetzt werden, krystallisiert es in Nadelbüscheln, die 1 Molekül Krystallwasser enthalten. Der Schmelzpunkt der reinen getrockneten Substanz liegt bei 298 bis $299^{\circ}$ (unkorr.), indem bei $292^{\circ}$ schwaches Sintern eintritt. In kalter Schwefelsäure löst sich die Substanz unter Orangefärbung. Die beim Erwärmen rotorange werdende Lösung zeigt beim Schütteln in dünner Schichte violette Färbung. Die Lösung fluoresziert gelb. Bei der Liebermann'schen Cholestolprobe wurden im Laufe einer Stunde folgende Färbungen beobachtet: violett, blauviolett, blau, blaugrün, dann grün, wobei die Lösung gelb fluoresziert. Bei der Salkowsky-Hesse'schen Probe färbt sich. die Schwefelsäure orangerot, dann rot mit gelber Fluoreszenz, das Chloroform färbt sich hellviolett.

In heißer, verdünnter Natronlauge, Sodalösung und Ammoniak löst sich das Resinol leicht auf. Beim Versetzen der erkalteten alkalischen Lösungen mit wenig Äther krystallisieren die Alkalisalze aus.

Die krystallisierte Substanz verliert ihr Krystallwasser sehr schwer. Der durch Trocknen im Vakuum der Wasserstrahlpumpe ermittelte $z u$ hohe Gewichtsverlust dürfte durch geringe Mengen wegsublimierender Substanz bedingt sein.

Analysen der lufttrockenen Substanz:

1. $4 \cdot 280 \mathrm{mg}$ Substanz gaben $11 \cdot 51 \mathrm{mg} \mathrm{CO}_{2}$ und $3 \cdot 86 \mathrm{mg} \mathrm{H}_{2} \mathrm{O}$.

2. $4.299 \mathrm{ng}$ Substanz verloren beim Trocknen im Vakuum bei $125^{\circ}$ $0.212 \mathrm{mg}$.

3. $4 \cdot 266 m g$ Substanz verloren beim Trocknen im Vakuum bei $140^{\circ}$ $0 \cdot 210 \mathrm{mg}$.

In 100 Teilen:

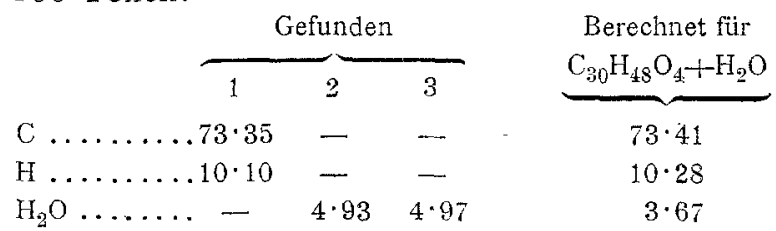

Analysen der getrockneten Substanz:

1. $4 \cdot 087 \mathrm{mg}$ Substanz gaben $11.41 \mathrm{mg} \mathrm{CO}_{2}$.

2. $4.016 m g$ Substanz gaben $11 \cdot 22 m g \mathrm{CO}_{2}$ und $3 \cdot 64 m g \mathrm{H}_{2} \mathrm{O}$.

3. $4.033 m g$ Substanz gaben $11.265 n g \mathrm{CO}_{2}$ und $3 \cdot 62 m_{\mathrm{S}} \mathrm{H}_{2} \mathrm{O}$. 
In 100 Teilen:

\begin{tabular}{|c|c|c|c|c|}
\hline & \multicolumn{3}{|c|}{ Gefunden } & \multirow{2}{*}{$\begin{array}{c}\begin{array}{c}\text { Berechnet für } \\
\mathrm{C}_{30} \mathrm{H}_{48} \mathrm{O}_{4}(472 \cdot 4)\end{array} \\
\end{array}$} \\
\hline & 1 & 2 & 3 & \\
\hline$c \ldots \ldots \ldots$ & $76 \cdot 14$ & $76 \cdot 20$ & $76 \cdot 18$ & $76 \cdot 21$ \\
\hline $\mathrm{H} \ldots \ldots \ldots$ & $\longrightarrow$ & $10 \cdot 15$ & $10 \cdot 04$ & $10 \cdot 24$ \\
\hline
\end{tabular}

Bestimmung der spezifischen Drehung im Mikropolarisationsapparat nach Emil Fischer (Lösungsmittel abs. Äthylalkohol):

$$
\begin{gathered}
p=2.061 \\
d=0.7994 \\
\mathcal{c}=1.647 \\
l=100 \mathrm{~mm} \\
l=50 \mathrm{~mm} \\
x_{D}^{9 \circ}=+0.85^{\circ} \quad \alpha_{D}^{9 \circ}=+0.42^{\circ} \text { bis }+0.43^{\circ} \\
{[\alpha]_{D}^{9 \circ}=+51.60^{\circ} .}
\end{gathered}
$$

Mikromolekulargewichtsbestimmung nach der Siedepunktsmethode:

Auch hier zeigte sich, daß bei Verwendung von Aceton als Lösungsmittel die erhaltenen Werte $\mathrm{zu}$ hoch ausfallen, offenbar durch den Einfluß der freien Hydroxylgruppe. Die Verwendung von Chloroform machte die schwere Löslichkeit der Substanz unmöglich. Durch graphische Extrapolation konnten Werte erhalten werden, die sich dem wahren Molekulargewichte nähern.

Bei der Molekulargewichtsbestimmung des Äthyläthers in Chloroform wurden sofort sehr gut stimmende Werte erhalten.

Lösungsmittel: Aceton 1.20 g.

$$
\begin{array}{lll}
\text { a) } s_{1}=6.93 \mathrm{mg} ; & \Delta_{1}=0.010^{\circ} ; & M_{1}=964 \\
s_{2}=15.65 \mathrm{mg} ; & \Delta_{2}=0.017^{\circ} ; & M_{2}=1281 \\
\text { b) } \quad s_{1}=8.10 \mathrm{mg} ; & \Delta_{1}=0.012^{\circ} ; & M_{1}=939 \\
s_{2}=14.11 \mathrm{mg} ; & \Delta_{2}=0.018^{\circ} ; & M_{2}=1094
\end{array}
$$

Die graphische Extrapolation ergibt

$$
\begin{aligned}
& a=715, \\
& b=725 .
\end{aligned}
$$




\section{d-Sumaresinolmethyläther.}

$d$-Sumaresinol wird in Ammoniak gelöst, der Überschuß an Ammoniak am Wasserbade möglichst vertrieben und mit Silbernitratlösung das Silbersalz ausgefällt. Das in voluminösen weißen Flocken abgeschiedene Saiz wird abgesaugt, gut mit Wasser gewaschen und im Vakuum über Schwefelsäure getrocknet.

Das trockene Silbersalz wird zur Darstellung des Äthers mit Jodmethyl und Methylalkohol eine Stunde am Wasserbade gekocht. Dann wird vom abgeschiedenen Silberjodid filtriert und die Lösung freiwillig eindunsten gelassen. Der Äther scheidet sich noch durch Jod verunreinigt in warzigen Krystallen ab. ${ }^{1}$ Zur weiteren Reinigung krystallisiert man ihn aus heißem Methylalkohol durch Zufügen von Wasser bis zur Trübung um. Die reine Verbindung schmilzt bei 215 bis $216^{\circ}$ (unkorr.), indem sie bei $212^{\circ}$ schwach zusammensintert.

Analysen der bei $110^{\circ}$ getrockneten Substanz:

1. 4.172. $\mathrm{mg}_{\mathrm{g}}$ Substanz gaben $11 \cdot 69 \mathrm{mg}^{\circ} \mathrm{CO}_{2}$ und $3 \cdot 85 \mathrm{mg} \mathrm{H}_{2} \mathrm{O}$.

2. $3.670 \mathrm{mg}$ Substanz gaben $1.71 \mathrm{mg} \mathrm{AgJ}$.

In 100 Teilen:

\begin{tabular}{|c|c|c|}
\hline - & Gefunden & $\begin{array}{c}\text { Berechnet für } \\
\mathrm{C}_{80} \mathrm{H}_{47} \mathrm{O}_{4} \mathrm{CH}_{3}(486 \cdot 4)\end{array}$ \\
\hline$c \ldots \ldots \ldots$ & $76 \cdot 42$ & $76 \cdot 48$ \\
\hline$H \ldots \ldots \ldots$ & $10 \cdot 33$ & $10 \cdot 36$ \\
\hline $\mathrm{O} . \mathrm{CH}_{3} \ldots \ldots$ & $6 \cdot 16$ & $6 \cdot 23$ \\
\hline
\end{tabular}

\section{d-Sumaresinoläthyläther.}

Diese Verbindung erhält man durch Kochen des Silbersalzes mit Jodäthyl und Äthylalkohol und Weiterbehandlung des Reaktionsproduktes wie beim Methyläther. Der Äther krystallisiert aus verdünntem Äthylalkohol in prachtvollen, langen,

1 Lidy (a. a. O., p. 67) reinigt auf umständlichem Wege seinen durch Jod verunreinigten Äther durch Lmfällen aus alkoholischer Lösung. Das tibrigens schon durch bloßes Lmkrystallisieren des Äthers aus verdinntem Methylalkohol leicht $z u$ beseitigende Jod kann auch einfach durch Ausschütteln der ätherischen Lösung des Athers mit verüinnter Natronlauge entfernt werden. 
weißen Nadeln vom Schmelzpunkt 207 bis $208^{\circ}$ (unkorr.) $\left(205^{\circ}\right.$ schwaches Sintern). Er ist in den meisten organischen Lösungsmitteln leicht löslich.

Analysen der bei $110^{\circ}$ getrockneten Substanz:

1. 4.120 mg Substanz gaben $11.57 \mathrm{mg} \mathrm{CO}_{2}$ und $3 \cdot 84 \mathrm{mg} \mathrm{H}_{2} \mathrm{O}$.

2. $3 \cdot 464 \mathrm{mg}$ Substanz gaben $1 \cdot 66 \mathrm{mg}$ AgJ.

In 100 Teilen:

\begin{tabular}{|c|c|c|}
\hline & - Gefunden & $\begin{array}{l}\text { Berechnet für } \\
\mathrm{C}_{30} \mathrm{H}_{45} \mathrm{O}_{4} \cdot \mathrm{C}_{2} \mathrm{H}_{5}\end{array}$ \\
\hline$\ldots \ldots \ldots$ & $76 \cdot 59$ & $76 \cdot 74$ \\
\hline $\mathrm{H}, \ldots \ldots \ldots$ & $10 \cdot 43$ & $10 \cdot 47$ \\
\hline O. $\mathrm{C}_{2} \mathrm{H}_{5}$ & $9 \cdot 19$ & $9 \cdot 00$ \\
\hline
\end{tabular}

Mikromolekulargewichtsbestimmung nach der Siedepunktsmethode:

Lösungsmittel: Chloroform $2 \cdot 25 \mathrm{~g}$.

$$
\begin{array}{ll}
s_{1}=10.33 \mathrm{mg} ; & \Delta_{1}=0.034^{\circ} ; \quad M_{1}=494 \\
s_{2}=19.85 \mathrm{mg} ; & \Delta_{2}=0.062^{\circ} ; \quad M_{2}=521 .
\end{array}
$$

Berechnetes Molekulargewicht für $\mathrm{C}_{32} \mathrm{H}_{52} \mathrm{O}_{4}=500 \cdot 4$. 